\title{
Systematic Identification of Hepatocellular Proteins Interacting with NS5A of the Hepatitis C Virus
}

\author{
Jiwon Ahn", Kyung-Sook Chung \\ Miyoung Nam, Shin-Jung Choi, Hyoung-Chin Kim, Michung Yoon \\ Suhn-Kee Chae ${ }^{*}$ and Kwang-Lae Ho** \\ Korea Research Institute of Bioscience and Biotechnology (KRIBB), Yusong, Daejeon, Korea \\ ${ }^{\S}$ Department of Biology, Mokwon University, Daejeon, Korea \\ "Department of Biochemistry, Paichai University, Daejeon, Korea
}

Received 16 June 2004, Accepted 23 August 2004

The hepatitis $\mathrm{C}$ virus is associated with the development of liver cirrhosis and hepatocellular carcinomas. Among the 10 polyproteins produced by the virus, no function has been clearly assigned to the non-structural 5A (NS5A) protein. This study was designed to identify the hepatocellular proteins that interact with NS5A of the HCV. Yeast twohybrid experiments were performed with a human liver cDNA prey-library, using five different NS5A derivatives as baits, the full-length NS5A (NS5A-F, amino acid (aa) 1 447) and its four different derivatives, denoted as NS5A-A (aa 1 150), -B (aa 1 300), -C (aa 300 447) and D (aa 150 447). NS5A-F, NS5A-B and NS5A-C gave two, two and 10 candidate clones, respectively, including an AHNAK-related protein, the secreted frizzled-related protein 4 (SFRP4), the $\mathrm{N}$-myc downstream regulated gene 1 (NDRG1), the cellular retinoic acid binding protein 1 (CRABP-1), ferritin heavy chain (FTH1), translokin, tumor-associated calcium signal transducer 2 (TACSTD2), phosphatidylinositol 4-kinase (PI4K) and centaurin 2 (CENT82). However, NS5A-A produced no candidates and NS5A-D was not suitable as bait due to transcriptional activity. Based on an in vitro binding assay, CRABP-1, PI4K, CENT 82 and two unknown fusion proteins with maltose binding protein (MBP), were confirmed to interact with the glutathione $S$-transferase (GST)/NS5A fusion protein. Furthermore, the interactions of CRABP-1, PI4K and CENTS2 were not related to the PXXP motif (class II), as judged by a domain analysis. While

'Present address: College of Oriental Medicine, Taejon University, Daejeon, Korea

These two authors contributed equally to this work.

*To whom correspondence should be addressed.

Tel: 82-42-860-4158; Fax: 82-42-860-4597

E-mail: kwanghoe@kribb.re.kr their biological relevance is under investigation, the results contribute to a better understanding of the possible role of NS5A in hepatocellular signaling pathways.

Keywords: HCV, NS5A, PXXP domain, Yeast two-hybrid

\section{Introduction}

The hepatitis $\mathrm{C}$ virus (HCV) is the major etiologic agent of non-A and non-B hepatitis (Kim, 2002). Chronic infection with $\mathrm{HCV}$ results in the development of liver cirrhosis and hepatocellular carcinomas (Tong et al., 1995). HCV belongs to the Flaviviridae family, having a $9.5-\mathrm{kb}$ positive-sense RNA genome (Choo et al., 1989). The RNA encodes a polyprotein (3,010 amino acids) in the following gene order: $\mathrm{NH}_{2}$-Core-E1-E2-p7-NS2-NS3-NS4A-NS4B-NS5A-NS5B$\mathrm{COOH}$ (Grakoui et al., 1993). Processing of the precursor polyprotein requires both host and viral proteases to produce the structural (core, E1, E2 and p7) and non-structural (NS2, NS3/4A, NS4B, NS5A and NS5B) proteins of the virion (Bartenschlager and Lohmann, 2000). The non-structural NS5A protein is generated in the form of a mature protein by the action of NS3/4A serine-protease.

NS5A has generated considerable interest because it interacts with a wide variety of host signaling proteins and, as such, may play a role in immune invasion and carcinogenesis (Street et al., 2004). One indication that NS5A may play a role in cell signaling is the presence of polyproline (PXXP) motifs, which are highly conserved throughout a range of $\mathrm{HCV}$ genotypes. Of particular interest is the observation that the highly conserved C-terminal PXXP polyproline motif in NS5A is able to interact with the Src-homology (SH) 3 domains of the adaptor protein, Grb2 (growth factor receptor- 
bound protein 2), and members of the Src family of tyrosine kinases (Tan et al., 1999; Macdonald et al., 2004). In addition to these interactions, it has been reported that NS5A interacts with the Core protein of the HCV (Goh et al., 2001), p53 (Majumder et al., 2001), CDK1 (Arima et al., 2001), the SNF-2 related CBP activator protein (SRCAP) (Ghosh $e t$ al., 2000), karyopherin $\beta 3$ (Chung et al., 2000), P85 phosphatidylinositol 3-kinase (He et al., 2002), a SNARE-like protein (hVAP-33, a $33 \mathrm{kDa}$ human vesicle-associated membrane protein-associated protein) (Tu et al., 1999), apolipoproteins (Shi et al., 2002) and the TNF receptor-associated factor (TRAF) 2 (Park et al., 2002). The implications of these interactions are unclear, although they suggest that NS5A may play a role in the modulation of mitogenic and replicationrelated signaling pathways in $\mathrm{HCV}$-infected hepatocytes.

As a first step in a systematic search for cellular proteins that interact with NS5A, a yeast two-hybrid system was employed, using NS5A-derivatives as bait. In this study, for the first time, the interactions of 14 new hepatocellular proteins with NS5A are reported. Although their biological relevance is currently under investigation, the results will contribute to a better understanding of the possible role of NS5A in liver cirrhosis and hepatocellular carcinomas.

\section{Materials and Methods}

Yeast two-hybrid screening In search of host proteins that interact with HCV NS5A, a yeast two-hybrid screening was performed, where the entire or partial cDNA-coding region of NS5A (genotype 1b, generously donated by Dr. Soichiro Miura at National Defense Medical College in Japan) was fused in-frame with the Lex DNA binding domain into the pHybLex/Zeo vector from Invitrogen (San Diego, USA) as bait for screening a liver cDNA library. The yeast two-hybrid screening was carried out following the protocol recommended by the manufacturer (Invitrogen, Hybrid Hunter \#K5000-01) (Ahn et al., 2003). For the construction of the bait plasmid containing the full-length or partial cDNA of NS5A (NS5A-F, aa 1 447; NS5A-A, aa 1 150); NS5AB, aa 1 300; NS5A-C, aa 300 447; NS5A-D, aa 150 447), PCR was performed, with the primers indicated in Table 1, and cloned into $\mathrm{pHybLex/Zeo} \mathrm{using} \mathrm{the} \mathrm{linker} \mathrm{sites} \mathrm{at} \mathrm{both} \mathrm{ends,} \mathrm{as} \mathrm{shown} \mathrm{in}$ Fig. 1A. Before full-scale screening, autonomous activation of the reporter gene ( $\beta$-galactosidase) by the bait was assessed by a colony-lift filter assay, as described in the next section. The liver cDNA library in pYESTrp $(500 \mu \mathrm{g}$, \#A203-01) and the NS5A derivatives in $\mathrm{pHybLex} / \mathrm{Zeo}$ (each $500 \mu \mathrm{g}$ ) were cotransfected into the L40 yeast strain (MATa, his34200 trp1-901 leu2-3112 ade2 LYS2::(4lexAop-HIS3) URA3::(8lexAop-lacZ)GAL4), using the standard

Table 1. PCR primers used in this study

\begin{tabular}{|c|c|c|c|}
\hline Primer name & Primer sequence & Restriction site & Size (mer) \\
\hline \multicolumn{4}{|c|}{ A. PCR primers used in the construction of $\mathrm{pHybLex/Zeo-NS5A-F}$} \\
\hline NS5A(Full-F) & 5'-CGTCGACTGCCTCCGGCTCGTG-3' & Sal I & 22 \\
\hline NS5A(Full-R) & 5'-GTCGACTCAGCAGCAGACGAC-3' & Sal I & 21 \\
\hline \multicolumn{4}{|c|}{ 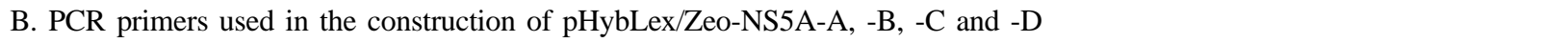 } \\
\hline NS5A (1-F) & 5'-GCGGCCGCTTTCCGGCTCGTGGCTAAGG-3' & Not $\mathrm{I}$ & 28 \\
\hline NS5A(150-F) & 5'-GCGGCCGCTTTTCACAGAATTGGATGGGGTGC-3' & Not $\mathrm{I}$ & 32 \\
\hline NS5A $(300-F)$ & 5'-GCGGCCGCTTGCGGAGATCCTGCGGAGAT-3' & Not I & 29 \\
\hline NS5A(150-R) & 5'-GTCGACTCATTAGAAGAATTCAGGGGCCGGAA-3' & Sal I & 32 \\
\hline NS5A(300-R) & 5'-GTCGACTCATTACGCCGGAACGGATACTTCCC-3' & Sal I & 32 \\
\hline NS5A(447-R) & 5'-GTCGACTCATTATCAGCAGCAGACGACGTCC-3' & Sal I & 31 \\
\hline \multicolumn{4}{|c|}{ C. PCR primers used in the construction of pMAL-cri/C14,- C18, -C39, -C45 and -C78 } \\
\hline $\mathrm{C} 14-\mathrm{F}$ & 5'-G GAATTCATGCCCAACTTCGCCGGCACCTG-3' & EcoRI & 30 \\
\hline $\mathrm{C} 14-\mathrm{R}$ & 5'-G GGATCCTCATTCCCGGACATAAATTCTGG-3' & BamHI & 30 \\
\hline $\mathrm{C} 18-\mathrm{F}$ & 5'-G GAATTCATGCGGCGTCTGCGGCCGCACCT-3' & EcoRI & 30 \\
\hline C18-R & 5'-G GGATCCTCATGGCGCTTCAGGGTCTCGCG-3' & BamHI & 30 \\
\hline $\mathrm{C} 39-\mathrm{F}$ & 5'-G GAATTCATGGATACACCGTTAAGGCGCAG-3' & EcoRI & 30 \\
\hline C39-R & 5'-G GGATCCTCAGATCTTGGCTGCCGGCTGCT-3' & BamHI & 30 \\
\hline $\mathrm{C} 45-\mathrm{F}$ & 5'-G GAATTCATGCAGAGTGCTGCAAAAGCCCC-3' & EcoRI & 30 \\
\hline $\mathrm{C} 45-\mathrm{R}$ & 5'-G GGATCCTCAGTAGGGGATGTCATTCTGAT-3' & BamHI & 30 \\
\hline $\mathrm{C} 78-\mathrm{F}$ & 5'-G GAATTCATGGGGGACACGCTGTCGGAGCA-3' & EcoRI & 30 \\
\hline $\mathrm{C} 78-\mathrm{R}$ & 5'-G GGATCCTCAGACGTTGCGCAGAAGAGACA-3' & BamHI & 30 \\
\hline \multicolumn{4}{|c|}{ D. PCR primers used in the construction of $\mathrm{pHybLex/Zeo-NS5A-F-mut} \mathrm{and} \mathrm{NS5A-C-mut}$} \\
\hline NS5A -mut1 & 5'-ATCGTCCTCTTCCTCCGTGCGGCTGCTATCGCAGCC & 'GGTG-3' & 49 \\
\hline NS5A mut2 & 5'-CACCTACCA AGGCCGCTGCGATAGCAGCCGCACGC & 'GACGAT-3' & 49 \\
\hline
\end{tabular}


A

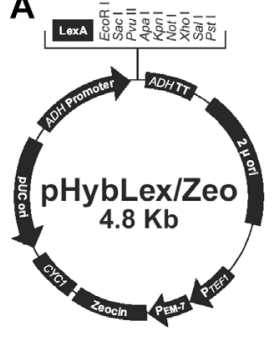

NS5A-F

NS5A-A

NS5A-B

NS5A-C

NS5A-D

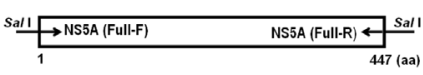

B

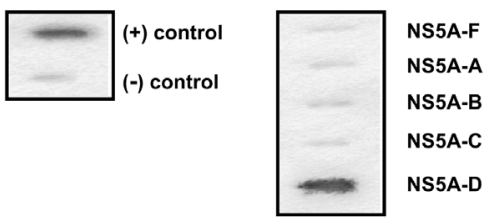

Fig. 1. Schematic drawings of the five different NS5A derivatives and a self-activation test. (A) The DNA fragments of the NS5A derivatives were PCR-amplified and subcloned into pHybLex/ Zeo, using the enzyme sites indicated at the flaking ends of the PCR products. (B) The five NS5A derivatives were tested to determine their suitability as bait for full-scale yeast two-hybrid screening by a colony-lift filter assay. For the (+) control, pHybLex/Zeo-Fos2 and pYESTrp-Jun were contransfected into the L40 yeast strain, and for the (-) control pHybLex/Zeo-Lamin was transfected alone. The NS5A-D type was eliminated from screening because it showed self-activated transcriptional activity.

lithium acetate method. At least $5 \times 10^{6}$ transformed cells were screened for growth on SD/Zeocin agar plates $(0.67 \%$ yeast nitrogen base without amino acid, $2 \%$ dextrose, $2 \%$ agar powder, 10X dropout solution without uracil, tryptophan, and histidine, supplements and $300 \mu \mathrm{g} / \mathrm{ml}$ Zeocin) over a 7-day period. Positive yeast transformants growing in the His-deficient medium were collected and replated for the $\beta$-galactosidase assay using the colony-lift filter procedure. For plasmid isolation, $\mathrm{His}^{+} / \mathrm{LacZ}^{+}$ positive colonies were grown, harvested and ruptured by vortexing with glass beads (mini-beadbeater). The pYESTrp plasmids containing the potential NS5A interacting cDNA inserts were

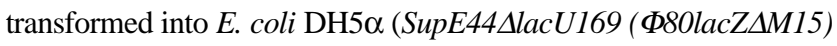
hsdR17 recAl endAl gyrA96 thi-1 relAl) and selected on LB/ ampicillin $(50 \mu \mathrm{g} / \mathrm{ml})$ plates. The plasmids were back-transformed into the L40 yeast strain and confirmed as $\mathrm{His}^{+} / \mathrm{LacZ}^{+}$positive. Positive interacting cDNA clones were analyzed by nucleotide sequencing and compared with known DNA or protein sequences deposited in GenBank using a BLAST search (http:// www.ncbi.nlm.nih.gov/ blast/).

Colony-lift filter assay For the colony-lift filter assay, yeast cells were grown on SD minimal plates, and transferred to a filter paper (Whatman no \#1). The filter was placed in liquid nitrogen for 30 sec, and incubated at room temperature in Z-buffer $(60 \mathrm{mM}$ $\mathrm{Na}_{2} \mathrm{HPO}_{4}, 40 \mathrm{mM} \mathrm{NaH} \mathrm{PO}_{4}, 10 \mathrm{mM} \mathrm{KCl}$ and $1 \mathrm{mM} \mathrm{MgSO}$ ), containing $0.82 \mathrm{mM}$ 5-bromo-4-chloro-3-indolyl- $\beta$ - $D$-galactopyranoside (X-Gal). The filters were placed at $30^{\circ} \mathrm{C}$ and monitored for color change as an indicator of the expressed $\beta$-galactosidase activity.
Construction of expression plasmids and protein expression For the construction of an expression plasmid producing the GST/ NS5A-F fusion protein, pGEX-4T-2, from Amersham Bioscience (Buckinghamshire, UK), was used as the parent plasmid. The DNA fragment of NS5A-F in pHybLex/Zeo was digested with Sal I, and transferred into the Sal I linker site of pGEX-4T-2 in frame. For the construction of the expression plasmids producing the MBP/C14, C18, C39, C45 and C78 fusion proteins, pMAL-cri (New England Biolabs, Beverly, USA) was used as the parent plasmid. The partial or entire cDNA of the five C-series clones were PCR-amplified with the primers (Bioneer, Daejeon, Korea) indicated in Table 1, digested with $E c o \mathrm{R} \mathrm{I}$ and $B a m \mathrm{H} \mathrm{I}$, and ligated into the $E c o \mathrm{R} \mathrm{I}$ Bam $\mathrm{H}$ I site of pMAL-cri. The resulting plasmids were transformed into E. coli DH5 $\alpha$ for expression. The expression of the GST/ NS5A and five MBP/C-series fusion proteins were induced by 0.5 $\mathrm{mM}$ isopropyl- $\beta$ - $D$-thiogalactopyranoside (IPTG) and $1 \mathrm{mM}$ maltose, respectively.

For the preparation of the fusion proteins, $100 \mathrm{ml}$ (MBP/C-series fusion proteins) or 1 liter (GST/NS5A-F fusion protein) volume cultures were harvested and resuspended in NETN buffer $(20 \mathrm{mM}$ Tris/Cl pH 8.0, $1 \mathrm{mM}$ EDTA, $100 \mathrm{mM} \mathrm{NaCl}$ and $0.5 \%$ (v/v) nonidet P (NP)-40), containing 10\% glycerol, $0.2 \%$ Tween-20, $1 \mathrm{mM} \beta$-mercaptoethanol and protease inhibitors $(1 \mathrm{mM}$ phenylmethylsulfonyl fluoride (PMSF), $1 \mu \mathrm{M}$ pepstatin, $1 \mu \mathrm{M}$ leupeptin and $10 \mathrm{nM}$ aprotinin). The cells were lysed by sonication, and the supernatants clarified by centrifugation at $4^{\circ} \mathrm{C}$. The expression of the GST/NS5A-F or MBP/C-series fusion proteins was analyzed by SDS-PAGE and Western blot analysis.

In vitro binding assay For the detection of $\mathrm{C}$-series proteins interacting with NS5A-F, a GST pull-down experiment was performed. Briefly, the supernatants, containing the GST/NS5A-F fusion protein, were immobilized on glutathione-Sepharose-4B beads (\#17-0756-01, Amersham Bioscience, Richmond, USA) for $1 \mathrm{~h}$ at $4^{\circ} \mathrm{C}$ on a rotating mixer, and the unbound GST/NS5A-F washed out with 10 volumes of NETN buffer by brief centrifugation. The supernatants containing the MBP/C-series fusion proteins were then allowed to interact with the immobilized GST/NS5A-F for $12 \mathrm{~h}$ at $4^{\circ} \mathrm{C}$. After 10 consecutive washes with 1 $\mathrm{ml}$ of NETN buffer containing $1 \%$ Triton X-100 and $0.5 \%$ NP-40, the beads were collected. Bound proteins were released by boiling in SDS sample buffer (62.5 mM Tris/Cl pH 6.8, 2\% SDS and 10\% glycerol), resolved by SDS-PAGE and transferred to membranes for Western blot analysis. A MBP pull-down experiment was performed vice versa, using amylose resin (\#800-21C) (New England Biolabs).

Western blot analysis Each sample was resolved by $10 \%$ SDSPAGE, transferred to PVDF membranes (\#66543, Pall, East Hills, USA), analyzed with antibodies, according to the suppliers protocol, and visualized with peroxidase and an enhancedchemiluminescence system (ECL Western blotting detection reagents \#RPN2106) from Amersham Bioscience (Hebert et al., 2003). The rabbit polyclonal GST (Z-5, $1: 2,000)$ and mouse MBP antibodies (MCA1412R, $1: 2,000)$ were purchased from Santa Cruz Biotechnology (Santa Cruz, USA) and SeroTec (Oxford, UK), respectively. The rabbit or mouse secondary antibodies, conjugated with horseradish peroxidase (NA934 or 
NA931, each $1: 5,000$ dilution), were purchased from Amersham Biosciences.

Mutation analysis For the construction of the pHybLex/ZeoNS5A-F-mut, where five proline amino acids of the PXXP motif (class II) were all mutated into alanine, two PCR reactions were performed with the pair of PCR primers (Bioneer); NS5A-mut1/ NS5A(447-R) and NS5A(1-F)/NS5A-mut2 (for sequences, refer to Table 1), using pHybLex/Zeo-NS5A-F as a template. A bloc PCR was then performed with the NS5A(1-F) and NS5A(447-R) primers, using the two PCR fragments as a template. The resulting PCR fragment was treated with Not I and Sal I, and ligated into the Not I/Sal I site of pHybLex/Zeo. For the construction of pHybLex/ Zeo-NS5A-C-mut, PCR was performed with the NS5A(300-F) and NS5A(477-R) primers. The PCR fragment was treated with Not I and Sal I, and ligated into the Not I/Sal I site of pHybLex/Zeo. For the interaction analysis, pHybLex/Zeo-NS5A-F-mut or pHybLex/ Zeo-NS5A-C-mut was cotransfected with pYESTrp-C45 and -C78, and analyzed by a colony-lift filter assay.

\section{Results}

Identification of hepatocellular proteins interacting with NS5A derivatives To identify the hepatocellular proteins that interacted with the HCV NS5A, a yeast two-hybrid system was exploited, with the human liver cDNA preylibrary, using NS5A derivatives as bait. As shown in Fig. 1A, five different NS5A derivatives were constructed as bait; the full-length NS5A (NS5A-F, aa 1 447) and its four different derivatives, denoted as NS5A-A (aa 1 150), -B (aa 1 300), C (aa 300 447) and D (aa 150 447). Of these, NS5A-D was not suitable as bait protein as it produced a blue color in the absence of a prey protein due to transcriptional activity (Fig. 1B) (Kato et al., 1997; Tanimoto et al., 1997).
At least $5 \times 10^{6}$ independent yeast colonies were screened for the four different NS5A derivatives, with the exception of NS5A-D. Primary selection for the $\mathrm{His}^{+} / \mathrm{LacZ}^{+}$phenotype resulted in $89,3,10$ and 82 positive clones for NS5A-F, NS5A-A, NS5A-B and NS5A-C, respectively. To further eliminate out-of-frame false positives, the cDNA inserts of the $\mathrm{His}^{+} / \mathrm{LacZ}^{+}$liver cDNA clones were sequenced and analyzed by the BLAST search program. As shown in Table 2, two, two and 10 candidate clones were finally identified as interacting with NS5A-F, NS5A-B and NS5A-C, respectively. Their cDNA inserts (aa) are represented, along with their entire open reading frame (ORF), in Table 2. Intriguingly, NS5A-A produced no candidate clones, even though it contained the proline-rich PXXP (class I) motif (Wientjes and Segal, 2003).

In vitro binding of the five $\mathrm{C}$-series cellular proteins with the full-length NS5A protein Out of the 14 new cellular proteins that interacted with NS5A, five C-series proteins (C14, C18, C39, C45 and C78) were selected and analyzed for their in vitro binding by means of a pull-down assay. The DNA fragments from NS5A-F and the five C-series clones were subcloned into pGEX-4T-2 and pMAL-cri in frame, as indicated in Figs. 2A and 2B, respectively. The NS5A and Cseries proteins were produced as GST and MBP fusion proteins in E. coli, and partially purified by single-step batch binding to glutathione-Sepharose4B beads and a maltoseamylose resin, respectively. The quality and quantity of the partially purified fusion proteins were assessed by Coomasie blue staining (Fig. 2C and 2D), and used for the next in vitro binding assay. The results showed that the fusion proteins had been successfully produced in a bacterial expression system.

For a pull-down experiment against the GST beads, bound proteins, through pull-down with GST beads, were eluted by

Table 2. List of cellular proteins interacting with NS5A-derivatives

\begin{tabular}{|c|c|c|c|c|}
\hline Clone No. & Gene & $\begin{array}{l}\text { Identified region / } \\
\text { entire ORF (aa) }\end{array}$ & GI & Accession No. \\
\hline F 82 & AHNAK-related protein & $71 \sim 293$ / 533 & 535176 & CAA52817 \\
\hline F 88 & SFRP4 (Secreted frizzled-related protein 4) & $69 \sim 315 / 346$ & 4506895 & NP_003005 \\
\hline В 01 & NDRG1 (N-myc downstream regulated gene 1 protein) & $123 \sim 261 / 394$ & 13112001 & AАH03174 \\
\hline В 03 & MGP (matrix Gla protein) & $1 \sim 103 / 103$ & 13528965 & ВC005272 \\
\hline C 14 & CRABP-I (Cellular Retinoic Acid Binding Protein 1) & $45 \sim 137 / 137$ & 4758051 & NM_004378 \\
\hline C 18 & Human Chromosome 19 Clone CTD-2537I9 & $1 \sim 280 / 315$ & 21743742 & AC008735 \\
\hline C 22 & FTH1 (Ferritin Heavy Chain) & $31 \sim 183 / 183$ & 16877183 & ВC016857 \\
\hline C 26 & Translokin & $57 \sim 500 / 500$ & 29469443 & AY225092 \\
\hline C 39 & Unknown (Protein for MGC 2574) & $4 \sim 294$ / 360 & 12655056 & ВC001378 \\
\hline C 44 & TACSTD2 (Tumor-associated calcium signal transducer 2) & $1 \sim 323$ / 323 & 14495611 & AАH09409 \\
\hline C 45 & PI4K (Phosphatidylinositol 4-kinase) & 1799 1916 / 2044 & 2326227 & AAD13352 \\
\hline C 66 & PTMA (Prothymosin $\alpha$, gene sequence 28) & $1 \sim 110 / 110$ & 18490876 & ВС022433 \\
\hline C 78 & CENT 22 (Centaurin $\delta 2$, Rho-GAP) & $850 \sim 1181 / 1209$ & 21264595 & NM_015242 \\
\hline C 80 & C9 ORF6 (Human chromosome 9 open reading frame 6) & 26 181 / 181 & 12002684 & AAG43367 \\
\hline
\end{tabular}


A

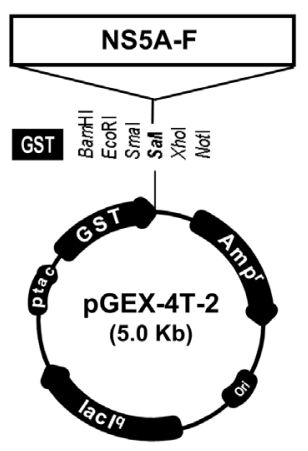

C

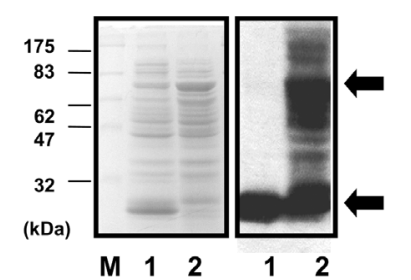

B

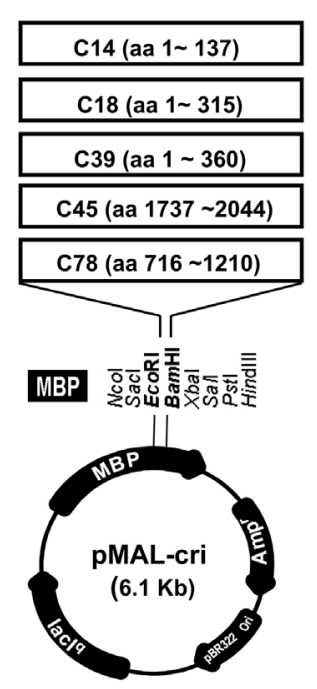

D

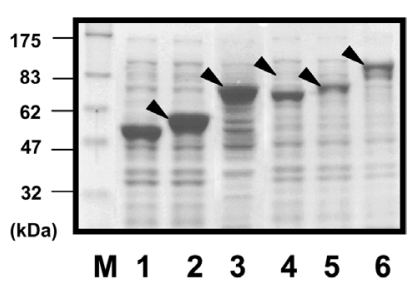

Fig. 2. Expression patterns of GST/NS5A-F and the five MBP/ $\mathrm{C}$-series fusion proteins. (A and B) NS5A-F and the five proteins that interact with NS5A-C were fused with GST and MBP, as illustrated. (C) The GST/NS5A-F fusion protein was produced in E. coli, resolved by SDS-PAGE, and subjected to Western blot analysis. Note: the GST/NS5A-F shows a band pattern consistent with degradation. The upper and lower arrows indicate the size of full-length GST/NS5A-F and GST only, respectively. Lane M: protein size marker; lane 1: GST only; lane 2: GST/NS5A-F. (D) The five $\mathrm{C}$-series proteins were fused with the MBP produced in E. coli and resolved by SDS-PAGE. The arrowheads indicate the expected molecular weight of each fusion protein. Lane $\mathrm{M}$ : protein size marker; lane 1: MBP only; lanes 2-6: MBP/C-14, C18, C-39, C-45 and C-78.

boiling, resolved by PAGE and analyzed by anti-GST and anti-MBP antibodies, as shown in Fig. 3. Similarly, a vice versa experiment against the MBP beads was performed. The results showed that NS5A directly interacted with the five different C-series clones, C14, C18, C39, C45 and C78.

Identification of the interacting domain The NS5A-C (aa 300 477), containing the PXXP motif (class II), functions as a potential region for interacting with many cellular proteins. Our results also showed that NS5A-C interacted with 10 different cellular proteins. Therefore, the issue of whether the PXXP motif was also important for their interactions with NS5A was also assessed. The proline amino acids of the PXXP motif (class II) were mutated to alanine by a PCR method, as illustrated in Fig. 4A, and whether the mutation

has an influence on the interactions was verified by a colony filter-lift assay. Two different clones, C45 (PI4K) and C78 (CENT82), were examined in relation to their possible interactions with the mutated NS5A-F and NS5A-C, as shown in Fig. 4B. According to the results, the mutation in the PXXP motifs did not affect their interactions with $\mathrm{C} 45$ and $\mathrm{C} 78$, suggesting they interacted with NS5A in a PXXP motifindependent manner. This finding indicates that the NS5A-C region has a novel domain, other than the $\mathrm{P} X X \mathrm{P}$ motif, which is responsible for the interactions.

\section{Discussion}

The lack of a robust in vitro replication system for HCV has meant the exact role of the NS5A protein in HCV infections remains unknown. To date, information concerning the potential NS5A function has derived, almost exclusively, from the use of a yeast two-hybrid system followed by an in vitro transient expression assay. A potential clue that NS5A may perturb other cellular signaling functions is the presence of several proline-rich PXXP motifs. Although NS5A contains multiple potential PXXP sites, only two are highly preserved among HCV genotypes, including both class I (aa 26 32) and class II (aa 350 -356) motifs. Such PXXP motifs bind to the $\mathrm{SH} 3$ domain found in a diverse group of signal-transducing molecules (Pawson, 1995). It has been well documented that the latter is necessary for NS5A to interact with Grb2, an adaptor protein consisting of one $\mathrm{SH} 2$ domain flanked by two SH3 domains. In accordance with these findings, NS5A-A (aa 1 150), which contains the class I PXXP motif, resulted in no candidate clones when used as bait protein in the yeast twohybrid system. In contrast, NS5A-C (aa 300 447), containing the class II PXXP motif, resulted in 10 interacting clones. However, these results do not mean that all these interactions arise from the PXXP motif, as judged by the results of the domain analysis showing that two of the tested clones interacted with NS5A through a novel domain(s) (Fig. 4B) and not the PXXP motif. Recent data from another group also suggests that the NS5A-B region has a novel binding site for interacting with the $\mathrm{SH} 3$ domain of PI3K p85 as well as the canonical PXXP motifs (Street et al., 2004).

It is conceivable that the role of the N-terminal region of NS5A is related with the regulation of NS5A itself, which masks the functional nuclear localization signal (aa 354 362), the class II PXXP motif (aa 350 356) and the transcriptional activating domain (aa 163 359) (Kato et al., 1997; Song et al., 2000). Consistent with this concept, NS5A-A (aa 1 150) resulted in no interacting candidates, with NS5A-D (aa 151 477) itself showing a blue color in the absence of a prey protein. According to previous reports, the N-terminally truncated form of NS5A functions as a potent transcriptional activator in both yeast and mammalian cells when fused to the DNA-binding domain of GAL4 (Kato et al., 1997; Tanimoto et al., 1997). Furthermore, the N-terminal deletion of NS5A, 


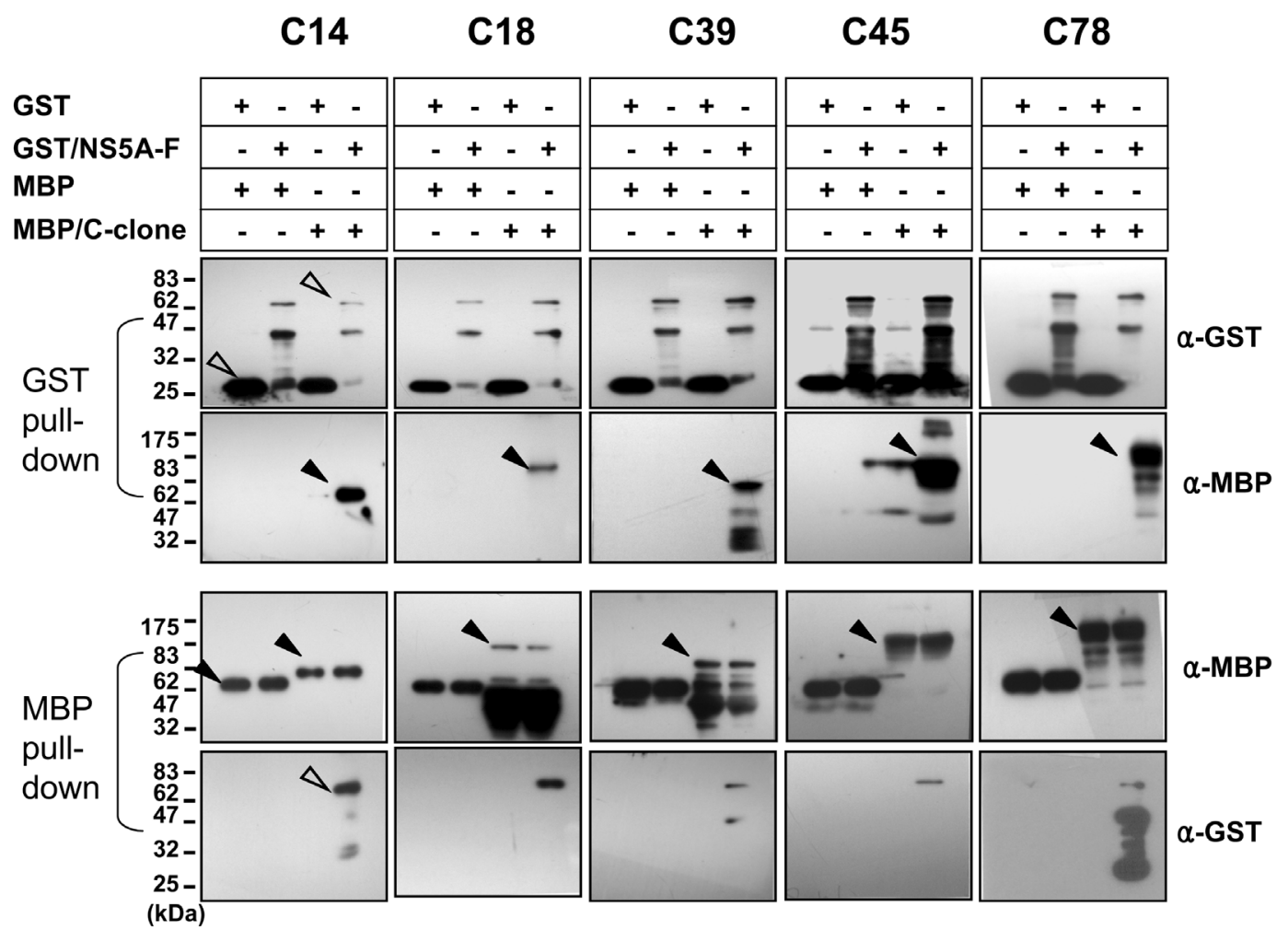

Fig. 3. In vitro interactions of NS5A-F with the five C-series proteins, C14, C18, C39, C45 and C78. GST/NS5A-F and each of the indicated MBP/C-series protein were mixed together and pulled-down by GST beads. The proteins were then resolved by SDS-PAGE and subjected to Western blot analyses with antibodies against GST and MBP (GST pull-down). The mixed proteins were pulled-down by MBP beads, and a vice versa Western blot analysis performed with antibodies against GST and MBP (MBP pull-down). The upper and lower arrowheads indicate the size of full-length GST/NS5A-F and GST only, respectively. The upper and lower filled arrowheads indicate the size of the MBP/C-series and MBP only, respectively.

by 66 aa or more, causes conformational alteration and exposes a cleavage site in the NS5A sequence around aa 150 (Song et al., 2000). Similarly, the bacterially produced GST/ NS5A-F fusion protein also caused the degradation of numerous discrete band patterns when observed by PAGE, suggesting that the N-terminal GST fusion resulted in the possible exposure of multiple cleavage sites and conformational alteration in the NS5A sequence (Fig. 2A). Therefore, it is granted that the number of clones interacting with NS5A-F or NS5A-B is less than that with NS5A-C, because the elimination of the masking $\mathrm{N}$-terminal region of NS5A exposures the highly interacting $\mathrm{C}$-terminal region. Therefore, the possibility of non-specific cellular interactions with the $\mathrm{N}$ terminally-deleted NS5A-C is not excluded. However, this is not the case because our results showed that the cellular interacting clones with NS5A-C also interact with NS5A-F, but with a slightly decreased affinity (data not shown).

The identified clones suggest a potential association with diverse cellular signaling pathways. For example, translokin (C26) has been reported to serve as an intracellular mediator of FGF-2 trafficking (Bossard et al., 2003), and PI4K (C45) is a well-known regulatory molecule of bioactive lipids in the regulation of cell motility, the Ras pathway, vesicle trafficking, secretion and apoptosis (Gehrmann and Heilmeyer, 1998). Furthermore, CENT 2 (C78) is a GTPase-activating protein for ARF-dependent and Rho-like GTPase, which may modulate G-protein-dependent signaling pathways for membrane trafficking and cytoskeleton dynamics (Santy and Casanova, 2002). Taken together, it is likely that NS5A perturbs diverse cellular signaling pathways through interacting with those cellular proteins, as in the previously well reported case of ERK signaling (Macdonald et al., 2004). In addition, the interaction of NS5A with those cellular proteins might disturb the membrane dynamics favoring virus replication. In summary, our experiments led to the identification of many hepatocellular molecules that may be related to cellular signaling pathways. Therefore, these results contribute to a better understanding of the possible role of NS5A in hepatocellular signaling pathways.

Acknowledgments This work was supported by the " $21^{\text {st }}$ Century Frontier R\&D Program" and "International S\&T Cooperation Program" from the MOST in the Republic of Korea. Jiwon Ahn was supported by the Research Intern Program Fellowship from the Korean Science and Engineering Foundation (KOSEF). Drs. Kyung-Sook Chung, 
A

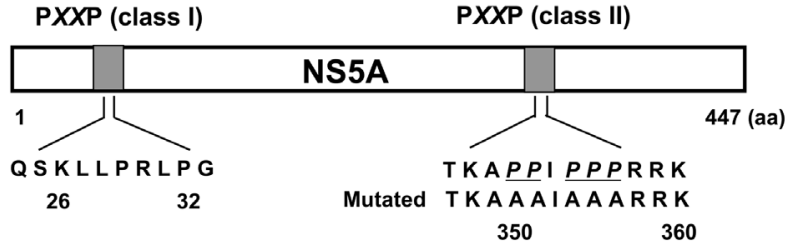

B

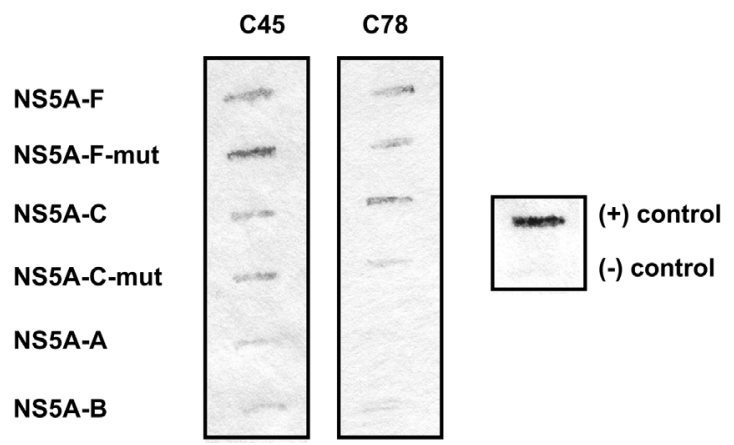

Fig. 4. Domain analysis for the interaction of NS5A-C with C45 and C-78. (A) The class II PXXP domain of NS5A-F or NS5A-C was mutated using a PCR method, as illustrated. (B) A Colony-lift filter assay was performed to detect whether pYES/ Trp-C-45 and -C-78 interact with the NS5A-F and NS5A-C mutants, where the proline amino acids of the PXXP motif are mutated to alanine. For the (+) control, pHybLex/Zeo-Fos2 and pYESTrp-Jun were contransfected into the L40 yeast strain, and for the (-) control pHybLex/Zeo-Lamin was transfected alone.

Dong-Uk Kim, Misun Won and Hyoung-Chin Kim were supported by the Intramural Research Program from KRIBB.

\section{References}

Ahn, B. H., Lee, J. H. and Bae, Y. S. (2003) Identification of mutations in protein kinase CKII $\beta$ subunit that affect its binding to ribosomal protein L41 and homodimerization. $J$. Biochem. Mol. Biol. 36, 344-348.

Arima, N., Kao, C. Y., Licht, T., Padmanabhan, R. and Sasaguri, Y. (2001) Modulation of cell growth by the hepatitis C virus nonstructural protein NS5A. J. Biol. Chem. 276, 12675-12684.

Bartenschlager, R. and Lohmann, V. (2000) Replication of the hepatitis C virus. Baillieres Best Pract. Res. Clin. Gastroenterol. 14, 241-254.

Bossard, C., Laurell, H., Van den Berghe, L., Meunier, S., Zanibellato, C. and Prats, H. (2003) Translokin is an intracellular mediator of FGF-2 trafficking. Nat. Cell. Biol. 5, 433-439.

Choo, Q. L., Kuo, G., Weiner, A. J., Overby, L. R., Bradley, D. W. and Houghton, M. (1989) Isolation of a cDNA clone derived from a blood-borne non-A, non-B viral hepatitis genome. Science 244, 359-362.

Chung, K. M., Lee, J., Kim, J. E., Song, O. K., Cho, S., Lim, J., Seedorf, M., Hahm, B. and Jang, S. K. (2000) Nonstructural protein $5 \mathrm{~A}$ of hepatitis $\mathrm{C}$ virus inhibits the function of karyopherin beta3. J. Virol. 74, 5233-5241.
Gehrmann, T. and Heilmeyer, L. M. Jr. (1998) Phosphatidylinositol 4-kinases. Eur. J. Biochem. 253, 357-370.

Ghosh, A. K., Majumder, M., Steele, R., Yaciuk, P., Chrivia, J., Ray, R. and Ray, R. B. (2000) Hepatitis C virus NS5A protein modulates transcription through a novel cellular transcription factor SRCAP. J. Biol. Chem. 275, 7184-7188.

Goh, P. Y., Tan, Y. J., Lim, S. P., Lim, S. G., Tan, Y. H. and Hong, W. J. (2001) The hepatitis C virus core protein interacts with NS5A and activates its caspase-mediated proteolytic cleavage. Virology 290, 224-236.

Grakoui, A., Wychowski, C., Lin, C., Feinstone, S. M. and Rice, C. M. (1993) Expression and identification of hepatitis C virus polyprotein cleavage products. J. Virol. 67, 1385-1395.

He, Y., Nakao, H., Tan, S. L., Polyak, S. J., Neddermann, P., Vijaysri, S., Jacobs, B. L. and Katze, M. G. (2002) Subversion of cell signaling pathways by hepatitis $\mathrm{C}$ virus nonstructural $5 \mathrm{~A}$ protein via interaction with Grb2 and P85 phosphatidylinositol 3-kinase. J. Virol. 76, 9207-9217.

Hebert, S., Berube, G. and Nepveu, A. (2003) Development of an in vitro assay for the proteolytic processing of the CDP/Cux transcription factor. J. Biochem. Mol. Biol. 36, 390-398.

Kato, N., Lan, K. H., Ono-Nita, S. K., Shiratori, Y. and Omata, M. (1997) Hepatitis C virus nonstructural region 5A protein is a potent transcriptional activator. J. Virol. 71, 8856-8859.

Kim, W. R. (2002) The burden of hepatitis C in the United States. Hepatology 36, 30-34.

Macdonald, A., Crowder, K., Street, A., McCormick, C. and Harris, M. (2004) The hepatitis C virus NS5A protein binds to members of the Src family of tyrosine kinases and regulates kinase activity. J. Gen. Virol. 85, 721-729.

Majumder, M., Ghosh, A. K., Steele, R., Ray, R. and Ray, R. B. (2001) Hepatitis C virus NS5A physically associates with p53 and regulates $\mathrm{p} 21 / \mathrm{waf} 1$ gene expression in a p53-dependent manner. J. Virol. 75, 1401-1407.

Park, K. J., Choi, S. H., Lee, S. Y., Hwang, S. B. and Lai, M. M. (2002) Nonstructural 5A protein of hepatitis $\mathrm{C}$ virus modulates tumor necrosis factor alpha-stimulated nuclear factor kappa B activation. J. Biol. Chem. 277, 13122-13128.

Pawson, T. (1995) Protein modules and signalling networks. Nature 373, 573-580.

Santy, L. C. and Casanova, J. E. (2002) GTPase signaling: bridging the GAP between ARF and Rho. Curr. Biol. 12, R360-362.

Shi, S. T., Polyak, S. J., Tu, H., Taylor, D. R., Gretch, D. R. and Lai, M. M. (2002) Hepatitis C virus NS5A colocalizes with the core protein on lipid droplets and interacts with apolipoproteins. Virology 292, 198-210.

Song, J., Nagano-Fujii, M., Wang, F., Florese, R., Fujita, T., Ishido, S. and Hotta, H. (2000) Nuclear localization and intramolecular cleavage of N-terminally deleted NS5A protein of hepatitis C virus. Virus Res. 69, 109-117.

Street, A., Macdonald, A., Crowder, K. and Harris, M. (2004) The Hepatitis C virus NS5A protein activates a phosphoinositide 3kinase-dependent survival signaling cascade. J. Biol. Chem. 279, 12232-12241.

Tan, S. L., Nakao, H., He, Y., Vijaysri, S., Neddermann, P., Jacobs, B. L., Mayer, B. J. and Katze, M. G. (1999) NS5A, a nonstructural protein of hepatitis $\mathrm{C}$ virus, binds growth factor receptor-bound protein 2 adaptor protein in a Src homology 3 domain/ligand-dependent manner and perturbs mitogenic 
signaling. Proc. Natl. Acad. Sci. USA 96, 5533-5538.

Tanimoto, A., Ide, Y., Arima, N., Sasaguri, Y. and Padmanabhan, R. (1997) The amino terminal deletion mutants of hepatitis C virus nonstructural protein NS5A function as transcriptional activators in yeast. Biochem. Biophys. Res. Commun. 236, 360364.

Tong, C. Y., Gilmore, I. T. and Hart, C. A. (1995) HCV-associated liver cancer. Lancet 345, 1058-1059.

Tu, H., Gao, L., Shi, S. T., Taylor, D. R., Yang, T., Mircheff, A. K., Wen, Y., Gorbalenya, A. E., Hwang, S. B. and Lai, M. M. (1999) Hepatitis C virus RNA polymerase and NS5A complex with a SNARE-like protein. Virology 263, 30-41.

Wientjes, F. B. and Segal, A. W. (2003) PX domain takes shape. Curr. Opin. Hematol. 10, 2-7. 